\title{
Targeting MKK3 as a novel anticancer strategy: molecular mechanisms and therapeutical implications
}

\author{
S Baldari ${ }^{1}$, V Ubertini ${ }^{1}, A$ Garufi ${ }^{1,2}$, G D’Orazi $^{*, 1,2}$ and G Bossi ${ }^{\star, 3}$
}

Mitogen-activated protein kinase kinase 3 (MAP2K3, MKK3) is a member of the dual specificity protein kinase group that belongs to the MAP kinase kinase family. This kinase is activated by mitogenic or stress-inducing stimuli and participates in the MAP kinase-mediated signaling cascade, leading to cell proliferation and survival. Several studies highlighted a critical role for MKK3 in tumor progression and invasion, and we previously identified MKK3 as transcriptional target of mutant (mut) p53 to sustain cell proliferation and survival, thus rendering MKK3 a promising target for anticancer therapies. Here, we found that targeting MKK3 with RNA interference, in both wild-type (wt) and mutp53-carrying cells, induced endoplasmic reticulum stress and autophagy that, respectively, contributed to stabilize wtp53 and degrade mutp53. MKK3 depletion reduced cancer cell proliferation and viability, whereas no significant effects were observed in normal cellular context. Noteworthy, MKK3 depletion in combination with chemotherapeutic agents increased tumor cell response to the drugs, in both wtp53 and mutp53 cancer cells, as demonstrated by enhanced poly (ADP-ribose) polymerase cleavage and reduced clonogenic ability in vitro. In addition, MKK3 depletion reduced tumor growth and improved biological response to chemotherapeutic in vivo. The overall results indicate MKK3 as a novel promising molecular target for the development of more efficient anticancer treatments in both wtp53- and mutp53-carrying tumors.

Cell Death and Disease (2015) 6, e1621; doi:10.1038/cddis.2014.591; published online 29 January 2015

MKK3 is a dual specificity protein kinase that belongs to the mitogen-activated protein kinase (MAPK) signaling pathway, an important signal transduction system that participates in a plethora of cellular programs, including cell differentiation, movement, division, and death. ${ }^{1}$ In particular, MKK3 is activated upon different forms of stressful stimuli and inflammatory cytokines ${ }^{2,3}$ through phosphorylation of serine and threonine residues at sites Ser189 and Thr193 ${ }^{4}$ by several upstream MAPK kinases, such as mixed lineage kinases, transforming growth factor-b-activated kinase 1 , and apoptosis signal-regulating kinase $1 .{ }^{5}$ Once activated, MKK3 specifically phosphorylates and activates p38MAPK at its activation site Thr-Gly-Tyr. ${ }^{2-4,6,7}$

Recent findings revealed that MKK3 has relevant role in tumor invasion and progression of gliomas and breast tumors. $^{8}$ Accordingly, we previously demonstrated that MKK3 is a novel upregulated target gene of mutant (mut)p53 gain-of-function activity, and that MKK3 knockdown strongly reduces cell proliferation and survival of mutp53bearing and p53-null human tumor cells. ${ }^{9}$ Interestingly, other studies demonstrated that MAPK14/p38MAPK is required for cell proliferation and survival, and that its inhibition leads to cell cycle arrest and autophagy-mediated cell death. ${ }^{10}$ Autophagy is an efficient degradation process that occurs at a basal rate in most cells, in which it helps to maintain homeostasis, acting as a cytoplasmic quality-control mechanism able to eliminate unnecessary, aggregating-prone proteins and injured organelles. ${ }^{11-15}$ Autophagy is also responsible for the survival response to growth-limiting conditions, such as nutrient deprivation. Under these stressful conditions, autophagy enhances its function as a survival mechanism, by which cellular components are sequestered into a doublemembrane vesicle, delivered to the lysosome system for final digestion ${ }^{16-19}$ and released for recycling of nutrients necessary to maintain protein synthesis, to produce substrates for oxidation and for ATP synthesis in the mitochondria ${ }^{20}$ and to contribute to the inhibition of apoptosis. ${ }^{21}$ However, nonproductive, uncontrolled, or prolonged autophagy leads to what has been designated 'autophagic cell death'.22,23 Several metabolic stresses can induce autophagy, such as hypoxia, oxidative stress, expression of aggregate-prone proteins, and glucose deprivation. ${ }^{24}$ An increasing number of studies also suggest that autophagy could be induced in consequence of the unfolded protein response, which is the major endoplasmic reticulum (ER) stress pathway. ${ }^{25}$ Indeed, ER stress stimulates autophagy through the PKR-like ER kinase (PERK)/eukaryotic translation initiation factor $2 a$ (elF2a) and Inositol-requiring Enzyme 1 (IRE1)/ c-Jun

\footnotetext{
${ }^{1}$ Experimental Oncology Laboratories, Regina Elena National Cancer Institute, Rome, Italy; ${ }^{2}$ Department of Medical, Oral and Biotechnological Sciences, University "G. d'Annunzio", Chieti, Italy and ${ }^{3}$ Laboratory of Medical Physics and Expert Systems, Regina Elena National Cancer Institute, Rome, Italy

*Corresponding author: G Bossi or G D'Orazi, Experimental Oncology Laboratories, Regina Elena National Cancer Institute, Via delle Messi d'Oro 156, Rome 00158, Italy; Tel: +39 0652662560; Fax: +39 0652662505; E-mail: bossi@ifo.it or gdorazi@unich.it

Abbreviations: MAP2K3, MKK3, mitogen-activated protein kinase kinase 3; Mut, mutant; Wt, wild type; ER, endoplasmic reticulum; PARP, poly (ADP-ribose) polymerase; PERK/elF2 $\alpha$, PKR-like ER kinase/eukaryotic translation initiation factor $2 \alpha$; IRE1, Inositol-requiring Enzyme 1; LC3, microtubule-associated protein 1 light chain 3; TET, tetracycline; DOX, doxycycline; sh/scr, short hairpin/scramble; CQ, Chloroquine; CHOP, CCAAT-enhancer-binding protein homologous protein; GRP78/Bip, glucoseregulated protein 78/immunoglobulin heavy chain-binding protein; ATG5, autophagy protein 5; ADR, adriamycin; 5-FU, 5-fluorouracil; MAPK mitogen-activated protein kinase

Received 22.10.14; revised 11.12.14; accepted 15.12.14; Edited by A Stephanou
} 
$\mathrm{N}$-terminal kinase 1 pathways. PERK/elF2a phosphorylation is essential for the transcription of key autophagy-associated genes during ER stress and may mediate the polyglutamineinduced microtubule-associated protein 1 light chain 3 (LC3) conversion, which is a marker of autophagy. ${ }^{26}$

Our previous studies suggested MKK3 as a general molecular player required to sustain cell proliferation and survival not only in mutp53-bearing but also in p53-null cancer lines. ${ }^{9}$ Here, we wanted to evaluate whether MKK3 played a role also in wild-type (wt) p53-bearing cells and its impact on both mup53 and wtp53 tumor cell response to anticancer drugs, investigating the molecular mechanisms involved in the biological outcomes upon MKK3 depletion. We found that MKK3 depletion reduced cell proliferation and survival of wtp53 cancer cells without affecting normal untransformed cells. Indeed, MKK3 depletion induced ER stress that correlated with stabilization and activation of wtp53. Moreover, MKK3 depletion induced cell autophagy that contributed to the degradation of mutp53, in agreement with recent studies. ${ }^{24,27}$ Furthermore, at biological level, MKK3 depletion in combination with chemotherapy reduces clonogenicity in both wtp53 and mutp53 cancer cells and induced higher in vivo anti-tumoral effects in a xenograft tumor model, when compared with drug treatment alone. The overall results revealed that, in the adopted in vitro and in vivo experimental tumor models, the MKK3 targeting might constitute an interesting strategy to improve anticancer treatment in both wtp53 and mutp53 cancer cells.

\section{Results}

MKK3 depletion reduces cell proliferation and viability in wtp53-bearing cancer but not normal cells. We previously showed that MKK3 is a general required factor to sustain cell proliferation and survival in mut- and null-p53 human cancer cell lines. ${ }^{9}$ Here, we aimed to explore whether MKK3 could have similar roles in wtp53 cell-context with a panel of human cancer (MFC7, HCT116) and primary nontransformed (FB1329, MCF10A) cell lines. All cell lines have engineered with conditional tetracycline (TET)-OFF lentiviralbased system carrying shRNA sequences specific to MKK3 (sh/MKK3) or RNA interference control (short hairpin/scramble (sh/scr)), and MKK3 depletion was obtained after treatment with TET analogous doxycycline (DOX), as previously described. ${ }^{9}$ We first studied the biological effects upon MKK3 depletion, in a time-dependent manner. Efficient MKK3 depletion (sh/MKK3) was achieved as early as $48 \mathrm{~h}$ upon DOX delivery in all tested cell lines, with respect to control cells (sh/scr), and maintained throughout time (Figures 1a-d, left panels). MKK3 depletion reduced cell proliferation and significantly increased cell death in both MCF7 and HCT116 cells (Figures 1a and b, middle and right panels). Interestingly, MKK3 depletion did not modify proliferation and viability of primary foreskin fibroblasts (FB1329) and normal mammary epithelial (MCF10A) cells (Figures 1c and d, middle and right panels).

Results suggest that MKK3 depletion exclusively hampers cell proliferation and viability of cancer but not normal untransformed cells.
MKK3 depletion stabilizes wtp53 protein. We next wanted to examine which molecular mechanisms could be involved in the biological outcome of MKK3 depletion. To this aim, we first analyzed whether MKK3 depletion could impact on wtp53 activity. We found that MKK3 depletion raised wtp53 protein levels in MCF7 and HCT116 cells (Figures 2a and b). The effect on wtp53 stabilization was likely at protein levels because p53 mRNA was not modified by MKK3 interference (Figure 2c). Of note, wtp53 stabilization upon MKK3 depletion correlated with induced expression of p21 at both protein (Figures $2 a$ and b) and mRNA (Figure 2c) levels. To investigate whether wtp53 is involved in the p21-induced gene expression, further assays were performed in double sh/MKK3 and sh/p53 knockdown cells. Figure $2 d$ shows that significant p21 expression occurred in p53-depleted cells, thus indicating that p53 is not responsible of p21 induction in MKK3 knockdown cells. This observation was further confirmed by similar experiments performed in $\mathrm{H} 1299$ p53-null cancer cells (Figure 2e).

MKK3 depletion induces autophagy and ER stress in wtp53 cancer cells. Microscopical examination revealed that MKK3 depletion induces the formation of large cytoplasmic vacuoles in cancer but not normal cells (data not shown). Because autophagic degeneration is always accompanied by cytoplasmic vacuolization, ${ }^{28}$ we asked whether MKK3 silencing might induce autophagy. To evaluate this mechanism, we analyzed microtubule-associated protein 1 LC3 conversion by western immunoblotting. Increased LC3 levels were observed in MCF7 and HCT116 cells upon MKK3 depletion, as evidenced by densitometric analyses of LC3-II/I ratio (Figure $3 a$ ). As autophagy is a dynamic process that begins with autophagosomes generation and terminates with their degradation in lysosomes, we investigated the autophagic flux by detecting the expression level of SQSTM1/ p62, a clear marker of autophagy being itself incorporated into the completed autophagosome and then degraded in autolysosomes. ${ }^{29}$ Marked p62 degradation was observed along with increased LC3-II levels after MKK3 depletion, indicating the existence of the autophagic flux (Figure 3a).

To further assess the autophagic flux in MKK3-depleted cells, confirmatory assays were performed with chloroquine (CQ), an inhibitor of the final stages of autophagy. ${ }^{30}$ As shown in Figure 3b, CQ induces higher accumulation of LC3-II and rescues p62 degradation in sh/MKK3 cells with respect to sh/scr-treated cells.

An increasing number of studies suggest that autophagy could be induced in consequence of the unfolded protein response, which is the major endoplasmic reticulum (ER) stress pathway. ${ }^{25}$ Here, we found that the mRNA level of CCAAT-enhancer-binding protein homologous protein (CHOP), a clear marker of ER stress ${ }^{31}$ was greatly increased after MKK3 depletion in both MCF7 and HCT116 cells (Figure 3c). In agreement with ER stress induction, MKK3 depletion increased the levels of glucose-regulated protein 78/immunoglobulin heavy chain-binding protein (GRP78/Bip) protein and induced elF2a phosphorylation (Figure 3d). The activation of ER stress pathway after MKK3 depletion correlated with wtp53 activation as confirmed by p53 phosphorylation at Ser392 (Figure 3d). Furthermore, we 


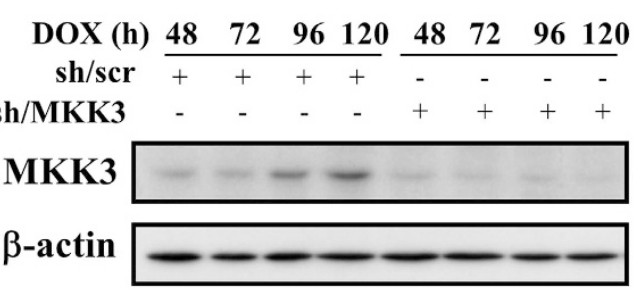

b

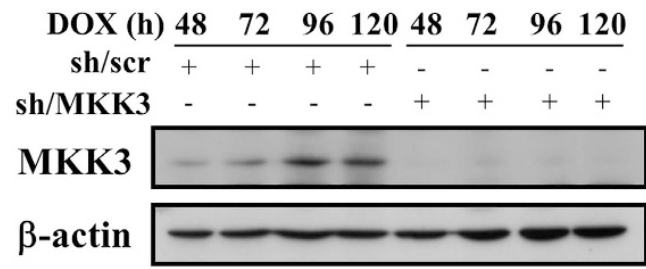

C

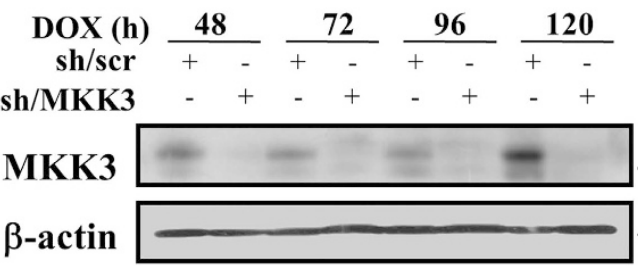

d

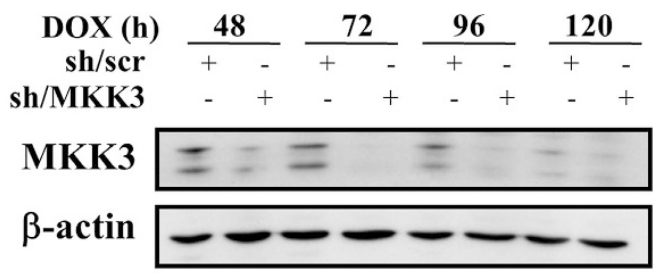

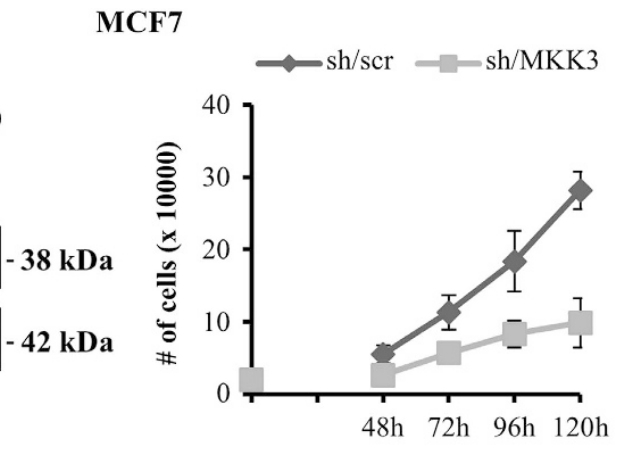

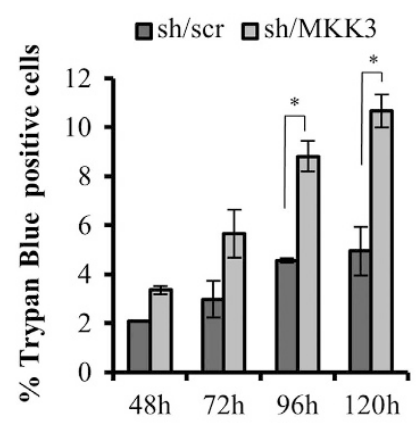

HCT116

-38 kDa

$-42 \mathrm{kDa}$
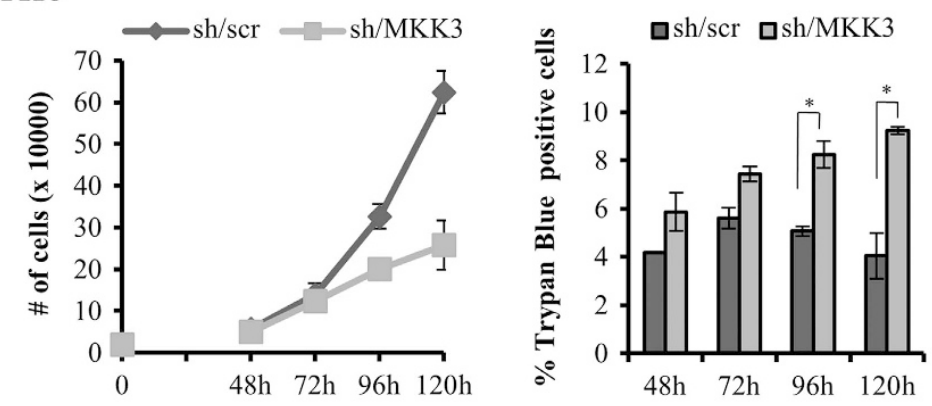

FB1329

$38 \mathrm{kDa}$
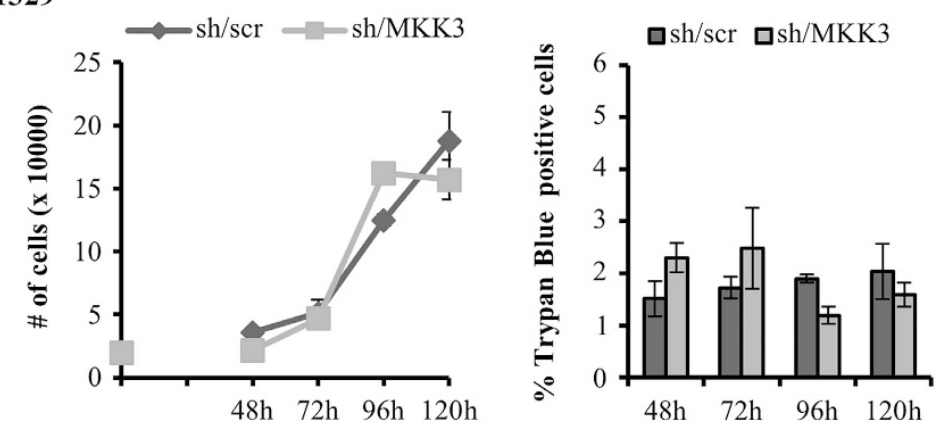

MCF10A

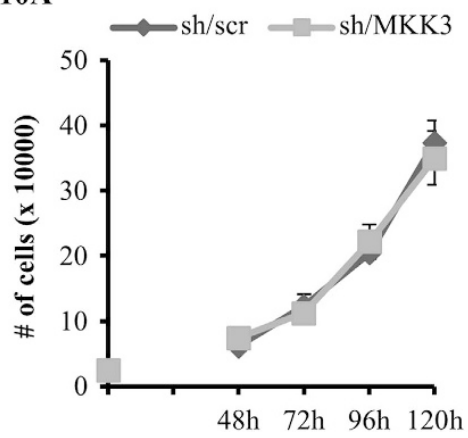

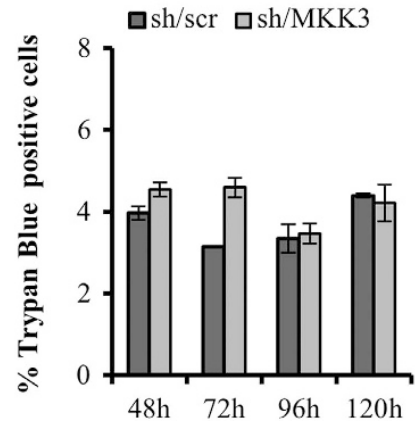

Figure 1 MKK3 depletion is detrimental to cancer but not normal cell proliferation and survival. Efficient MKK3 depletion was achieved in all tested lines (a-d, left panels): Engineered MCF7 (a), HCT116 (b), FB1329 (c) MCF10A (d) -sh/scr and -sh/MKK3 sublines (1.5 $\times 10^{5} \mathrm{celll} / 60 \mathrm{~mm}$ dish) were challenged with DOX (1.0 $\left.\mu \mathrm{g} / \mathrm{ml}\right)$ and cells collected time dependently $(48,72,96$ and $120 \mathrm{~h}$ ) to assess the MKK3 depletion efficiency. Then, protein lysates ( $30 \mu \mathrm{g} /$ /lane) were resolved in SDS-polyacrylamide gel electrophoresis and filter analyzed by western blot analysis with specific anti-MKK3 and anti- $\beta$-actin (loading control) antibodies. MKK3 depletion affects cell proliferation and viability of wtp53 tumor but not normal cells (a-d, right panels). Engineered MCF7 (a), HCT116 (b), FB1329 (c) MCF10A (d) -sh/scr and -sh/MKK3 sublines were seeded (2×104 cells/6-well plates) in DOX conditions. At the indicated time points, cells were harvested and quantified for viable and death cells by Trypan blue exclusion assays. Results are reported as means and S.D. of three independent experiments. ${ }^{*}$ The significance $(P<0.05)$ of death percent in sh/MKK3 with respect to sh/scr subline 
tested the impact of autophagy on cell viability by using $C Q$. As shown in Figure $3 e$, the increased cell death upon MKK3 depletion was significantly counteracted by blocking autophagy with $\mathrm{CQ}$.

To further confirm whether MKK3 depletion induces autophagic cell death, small RNA interference approaches were adopted to knockdown the essential autophagic gene ATG5, a member of the ATG family necessary for autophagosome elongation. ${ }^{32}$ Efficient autophagy protein 5 (ATG5) silencing rescues p62 degradation, as readout of autophagic process inhibition (Figure $3 \mathrm{f}$ ), and significantly reduces the apoptotic cell death in MKK3-depleted cells (Figure 3g), morphologically distinguished by severe chromatin condensation and nuclear fragmentation upon ethidium bromide cellular uptake, a marker of plasma membrane disruption.

Results suggest that MKK3 depletion triggers ER stress pathway and autophagic cell death in wtp53 cancer cells.

MKK3 depletion reduces mutp53 protein levels through autophagy. Recent studies disclosed the role of autophagy in mutp53 degradation ${ }^{24,27}$ and we above found that MKK3 depletion induced autophagy in wtp53 cells. Therefore, we

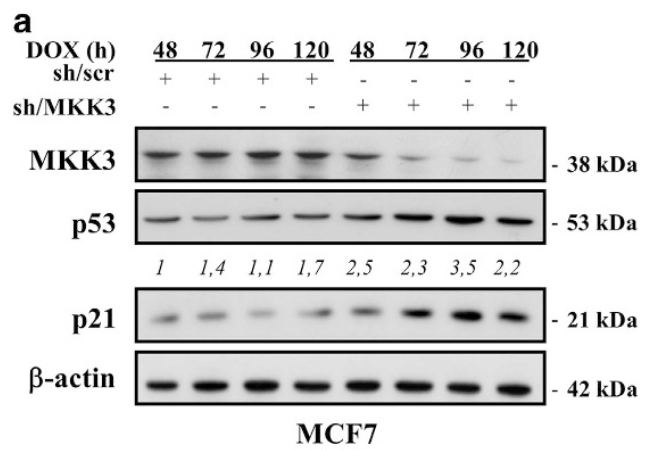

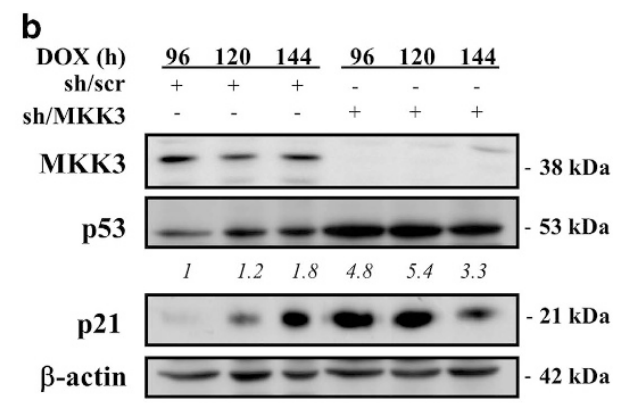

HCT116

d
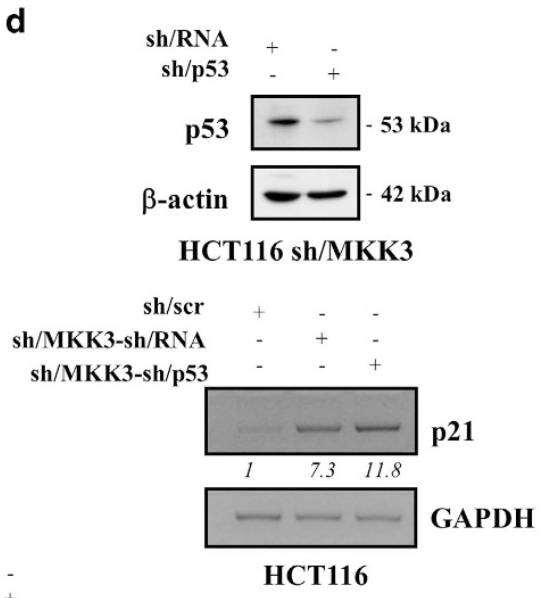

d
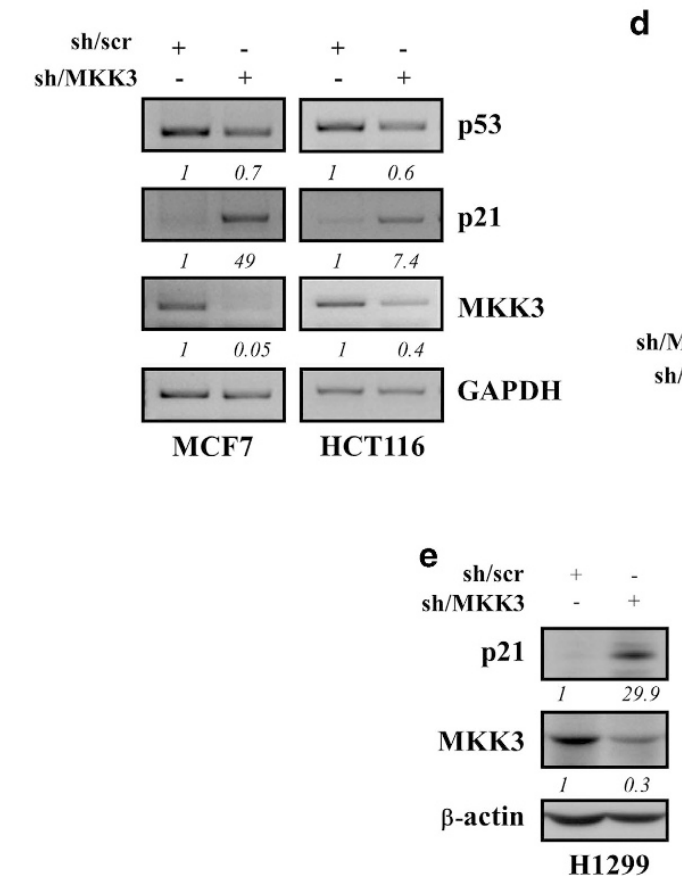
next aimed to test whether MKK3 knockdown could influence mutp53 levels and autophagy. We found that mutp53 protein levels underwent efficient reduction upon MKK3 depletion in both MDA-MB468 and HT29 cells (Figures 4a and b). Moreover, MKK3 depletion induced autophagy also in mutp53 cells, as well as above for wtp53 cells, as evidenced by LC3-II induction and p62 degradation (Figure 4c). Noteworthy, a significant delay in autophagy induction was observed in mutp53 cells upon MKK3 depletion when compared with wtp53 cells (120-144 h versus $72-96 \mathrm{~h}$, respectively), which correlates with mutp53 protein reduction.
Genetic approaches showed that ATG5 depletion rescues the p62 degradation in mutp53 sh/MKK3 cells further confirming autophagy (Figure 4d). Moreover, importantly, the use of autophagy inhibitor $\mathrm{CQ}$ efficiently rescued mutp53 protein levels in sh/MKK3 cells (Figure 4e), strongly suggesting that autophagy, induced upon MKK3 depletion, may have major roles in mutp53 protein reduction.

MKK3 depletion combined with chemotherapy decreases cell survival fractions and allows reducing dose in both wtp53 and mutp53 cancer cells. Based on the achieved a

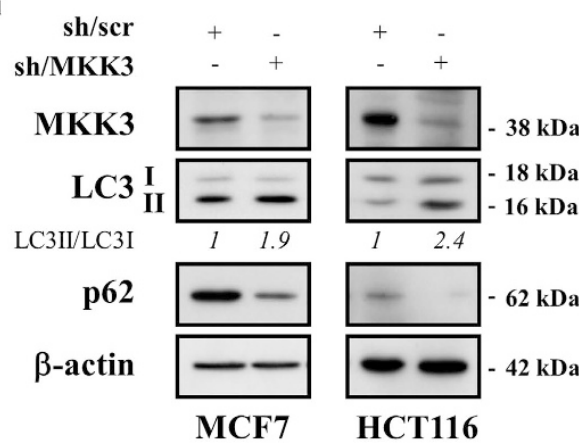

c

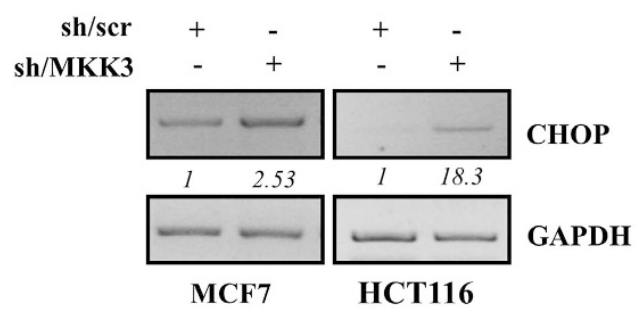

e

MCF7

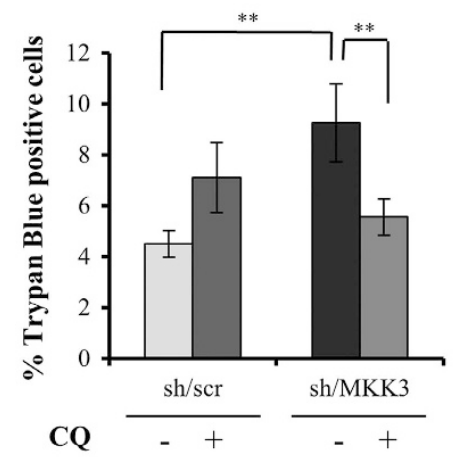

b

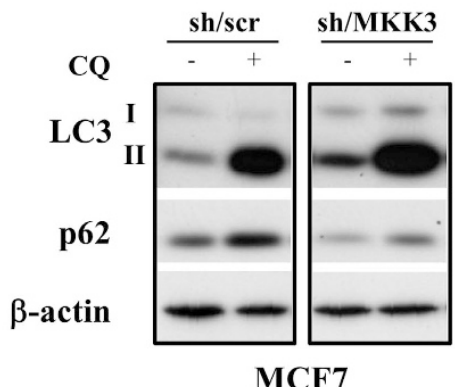

d

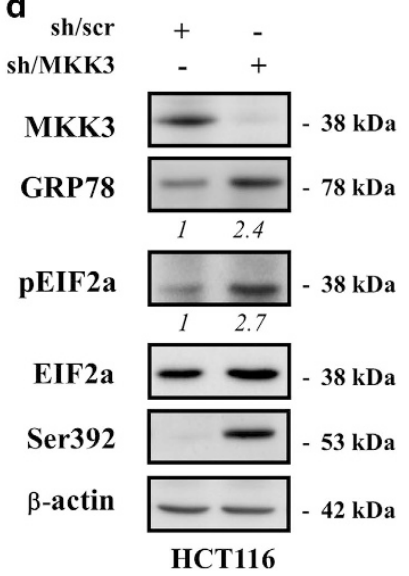

f

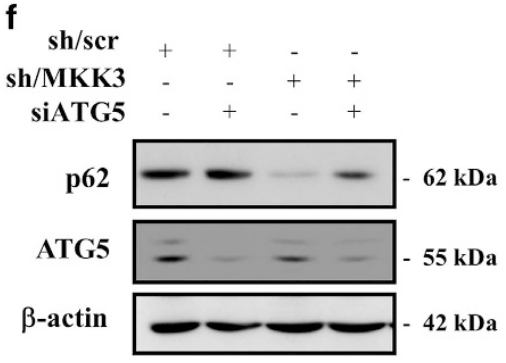

HCT116 g

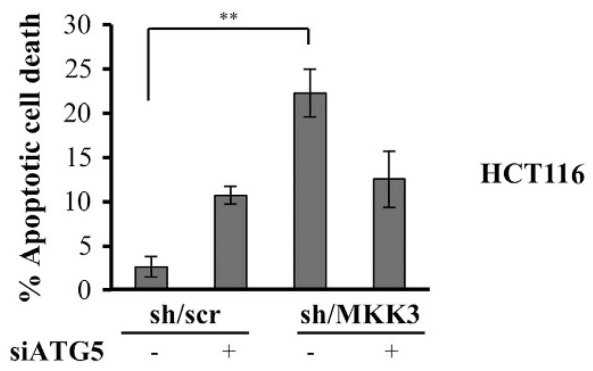


results, we investigated whether targeting MKK3 in combination with chemotherapy could improve therapeutic response. To this aim, the apoptotic response to different adriamycin (ADR) doses was analyzed in both wtp53 and mutp53 sh/MKK3 and sh/scr cells. As shown in Figure 5a, combined MKK3 knockdown with ADR treatment induced significantly higher poly (ADP-ribose) polymerase (PARP) cleavage in both wtp53 and mutp53 cells, with respect to ADR-treated control cells (sh/scr). Noteworthy, the lower dose of ADR in MKK3-depleted cells induces a significantly higher PARP cleavage with respect to control sh/scr cells challenged with the higher ADR dose with both wt and mutp53 cells (Figure 5a). Results are suggesting that MKK3 targeting combined to ADR treatment would provide a better therapeutic response allowing chemotherapeutic dose reduction in both wt and mutp53 cancer lines.

We next assessed long-term responses to ADR treatment by clonogenic assay. As shown in Figure $5 b$, the reduced clonogenic cell survival upon MKK3 depletion was markedly improved after ADR treatment, compared with ADR-treated control cells (sh/scr; Figure $5 \mathrm{~b}$ ), as also evidenced by densitometric analyses of clonogenic assays (Figure $5 \mathrm{c}$ ). These findings reveal a potential additive effect of MKK3 depletion on tumor cell response to drugs, thus pointing at MKK3 as a novel potential clinical target to improve both wtp53 and mutp53 cancer cell response to chemotherapeutic agents.

MKK3 depletion affects xenograft tumor growth and
potentiates chemotherapeutic effect in vivo. To evaluate
the MKK3 role in more physiological experimental models, we
explored in vitro and in vivo HT29 cancer cell response to
5 -fluorouracil (5-FU), a commonly used chemotherapeutic
drug in colon cancer patients. ${ }^{33}$ In accordance to the above
data with ADR, HT29 clonogenic cell survival was markedly
and dose-dependently reduced after 5-FU treatment,
compared with drug-treated control cells (sh/scr; Figure 6a).
Noteworthy, control sh/scr cells challenged with higher 5-FU
dose ( $10 \mu \mathrm{M}$ ) generated a number of colonies significantly
higher with respect to MKK3-depleted cells treated with the lower dose $(1.0 \mu \mathrm{M})$, further confirming the evidence that combined treatments allow chemotherapeutic dose reduction. Next, we generated tumor xenografts with HT29-sh/scr or -sh/MKK3 cells injected in nude mice, where MKK3 was efficiently reduced after DOX delivery (Figure $6 \mathrm{~b}$ ). In vivo results of tumor growth showed that MKK3 depletion per se significantly reduced tumor volume, compared with xenografts derived from control cells (sh/scr; Figure 6c, $P<0.05$ ); interestingly, MKK3 depletion further increased the effect of 5 -FU on tumor growth (Figure 6c, $P=0.01$ ), in agreement with our hypothesis that MKK3 targeting could constitute a novel therapeutic strategy to improve tumor response to therapies.

\section{Discussion}

MKK3 is a dual specificity protein kinase that belongs to the MAP kinase kinase family. This kinase is activated by mitogenic or stress-inducing stimuli and participates in the MAP kinase-mediated signaling cascade, leading to cell proliferation and survival. Recent findings suggested important roles for MKK3 in tumor invasion and progression. Accordingly, we previously identified MKK3 as a novel mutp53 target gene involved in tumor growth and survival in breast and colon cancer lines. ${ }^{9}$ In the present study, we wanted to evaluate whether MKK3 could play a role also in wtp53bearing cells. We found that MKK3 depletion strongly inhibited proliferation and survival of wtp53-bearing cancer cell lines, whereas it did not have any relevant effects on untransformed cells. In the attempt to identify the molecular mechanisms involved in such biological outcomes, we found that MKK3 depletion affected several pathways: (i) it triggered ER stress and autophagy, (ii) stabilized wtp53, and (iii) degraded mutp53.

Autophagy is a degradative process through which damaged organelles and misfolded proteins are targeted for disruption via the lysosomes. In cancer, autophagy may contribute to tumor cell survival. In established tumors, autophagy might act as a pro-survival pathway in response to metabolic stresses such as nutrient deprivation, hypoxia,

\footnotetext{
Figure 3 MKK3 depletion induces autophagic cell death and ER stress in wtp53 cancer cells. Markers of autophagy LC3-I, LC3-II, and p62 upon MKK3 depletion were assessed by western blotting (a) in wtp53 MCF7 (left panel) and HCT116 (right panel) engineered -sh/scr and sh/MKK3 sublines. Seeded cells $\left(1.5 \times 10^{5} \mathrm{cells} / 60 \mathrm{~mm}\right.$ dish) were collected at $72 \mathrm{~h}$ post DOX delivery, and protein lysates (15 $\mu \mathrm{g}$ /lane) resolved in SDS-polyacrylamide gel electrophoresis (PAGE) and probed with anti-MKK3-, anti-LC3-, and anti-SQSTM1/p62-specific antibodies. Densitometry analyses were performed with ImageJ software and LC3-II band intensity normalized to $\beta$-actin and quantified with respect to control tumors (sh/scr) set to 1.0. (b) MCF7-sh/scr and -sh/MKK3 sublines cultured in DOX condition (72 h) in the presence/absence of $25 \mu \mathrm{M} \mathrm{CQ}$ (48 h), then protein lysate were analyzed by western blot analysis with antibodies specific to LC3, SQSTM1/p62, and $\beta$ actin (loading control). (c) Engineered sh/MKK3 and sh/scr MCF7 and HCT116 cultured $48 \mathrm{~h}$ with DOX were collected and total RNAs analyzed by RT-PCR with set of primers specific to CHOP and GAPDH (housekeeping gene). Densitometry was performed with the ImageJ software and relative CHOP mRNA levels were normalized to GAPDH and quantified with respect to control tumors (sh-scr) set to 1.0. (d) Engineered sh/scr and sh/MKK3 HCT116 cell lines were maintained in DOX condition for $36 \mathrm{~h}$, then cells were collected and protein lysates ( $30 \mu \mathrm{g} / \mathrm{lane}$ ) analyzed by western blot for the presence of ER stress proteins: phosphorylated EIF2A protein was evaluated using phospho-specific antibodies. Total amount of EIF2A was determined using anti-EIF2A antibody. GRP78/Bip was also used as marker of ER stress. Ser392 was analyzed as p53 stabilization marker. $\beta$-Actin was used as loading control. Densitometry was performed with the ImageJ software and relative ER stress marker protein levels were normalized to actin and quantified with respect to control tumors (sh-scr) set to 1.0. (e) Autophagy inhibition rescues the cell death induced by MKK3 depletion. Engineered MCF7-sh/scr and -sh/MKK3 sublines were seeded $\left(1.5 \times 10^{5} \mathrm{cells} / 60 \mathrm{~mm}\right.$ dish) in DOX condition for $24 \mathrm{~h}$ then treated/ untreated with $\mathrm{CQ}(25 \mu \mathrm{M})$ and collected after $96 \mathrm{~h}$ of total DOX induction. Cell viability was evaluated with trypan blue exclusion assay. Results are reported as mean \pm S.D. of three independent experiments. Significance was assessed by Student's $t$-test, ${ }^{* *} P<0.01$. (f) Engineered HCT116-sh $/ \mathrm{scr}$ and -sh/MKK3 sublines were seeded (1.5 $\times 10^{5} \mathrm{cells} /$ $60 \mathrm{~mm}$ dish) and maintained in DOX condition for $48 \mathrm{~h}$, then transfected with siRNA for ATG5 (si-ATG5) or with control siRNA (si-ctr). Forty-eight hours after transfection, cells were collected and protein lysates ( $15 \mu \mathrm{g} / \mathrm{lane}$ ) resolved in SDS-PAGE and probed with anti-ATG5- and anti-SQSTM1/p62-specific antibodies. $\beta$-Actin was used as loading control. (g) Engineered HCT116-sh/scr and -sh/MKK3 sublines, treated as in $\mathrm{f}$, were collected after $96 \mathrm{~h}$ of DOX induction and then stained with DAPI and ethidium bromide and analyzed by fluorescent microscope. The number of apoptotic dead cells was calculated and reported as a percentage of the total number of cell counted. Results are reported as means and S.D. of three independent experiments. ${ }^{*}$ The significance $(P<0.01)$ of death percent in sh/MKK3 with respect to sh/scr subline
} 
a

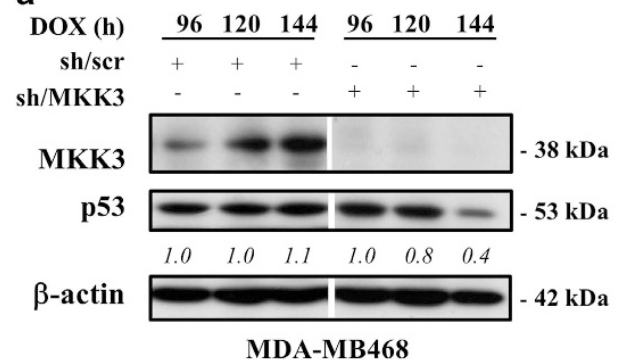

C

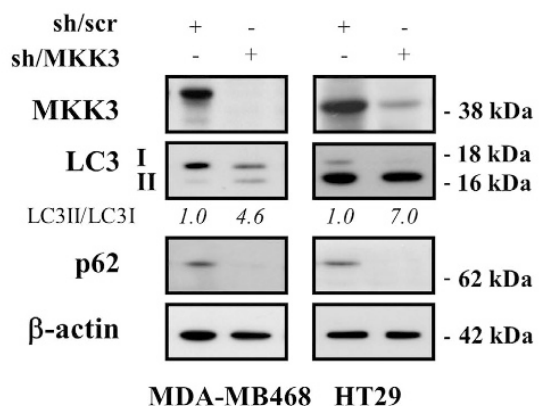

b

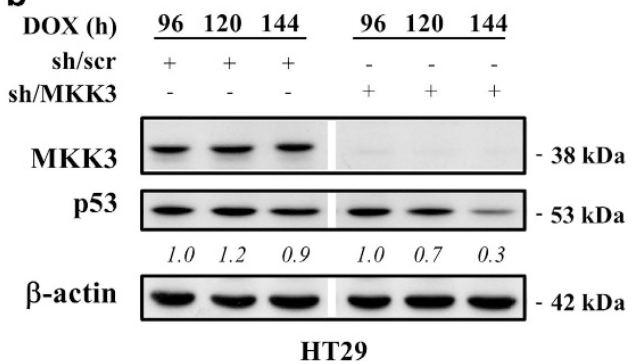

d

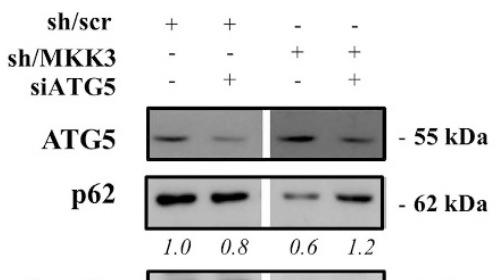

$\beta$-actin $-42 \mathrm{kDa}$

HT29

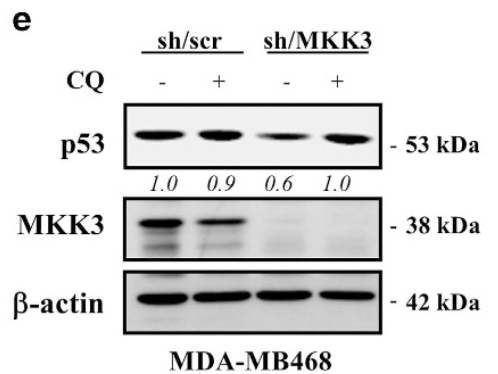

Figure 4 MKK3 depletion reduces mutp53 protein levels through autophagy. Engineered MDA-MB468 (a) and HT29 (b) -sh/scr and -sh/MKK3 sublines were cultured with DOX $(1.0 \mu \mathrm{g} / \mathrm{ml})$ and collected at indicated time points. Protein lysates (30 $\mu \mathrm{g} / \mathrm{lane})$ were analyzed by western blot analysis with anti-MKK3-, anti-p53-, and anti- $\beta$-actin (loading control)-specific antibodies. Densitometry was performed with ImageJ software and relative p53 band intensity normalized to $\beta$-actin and quantified with respect to controls (sh-scr) set to 1.0. (c) Markers of autophagy LC3-I, LC3-II, and p62 upon MKK3 depletion were assessed by western blotting in mutp53 MDA-MB468 (left panel) and HT29 (right panel) engineered -sh/scr and sh/MKK3 sublines. Seeded cells $\left(1.5 \times 10^{5} \mathrm{cells} / 60 \mathrm{~mm}\right.$ dish) were collected at $120 \mathrm{~h}$ post DOX delivery, and protein lysates (15 $\mu \mathrm{g} /$ lane) resolved in SDS-polyacrylamide gel electrophoresis (PAGE) and probed with anti-MKK3-, anti-LC3-, and anti-SQSTM1/p62-specific antibodies. Densitometry analyses were performed with ImageJ software and LC3-II band intensity normalized to $\beta$-actin and quantified with respect to control tumors (sh/scr) set to 1.0. (d) Engineered HT29-sh/scr and -sh/MKK3 sublines were seeded $\left(1.5 \times 10^{5}\right.$ cells/60 mm dish) and maintained in DOX condition for $72 \mathrm{~h}$, then transfected with siRNA for ATG5 (si-ATG5) or with control siRNA (si-ctr). Forty-eight hours after transfection, cells were collected and protein lysates (15 $\mu \mathrm{g} / \mathrm{lane})$ resolved in SDS-PAGE and probed with anti-ATG5- and anti-SQSTM1/ p62-specific antibodies. $\beta$-Actin was used as loading control. Densitometry analyses were performed with ImageJ software and anti-SQSTM1/p62 band intensity normalized to $\beta$ actin and quantified with respect to control tumors (sh/scr) set to 1.0. (e) MDA-MB468 sh/scr and sh/MKK3 sublines were cultured $120 \mathrm{~h}$ with DOX and, in the last $48 \mathrm{~h}$, treated/ untreated with CQ $(25 \mu \mathrm{M})$. Afterwards, cells were collected and lysate analyzed by western blot analysis with anti-p53-, anti-MKK3-, and anti- $\beta$ actin (loading control)-specific antibodies

absence of growth factors, and in the presence of chemotherapy or some targeted therapies that might mediate resistance to anticancer therapies. ${ }^{34-36}$ However, persistent or excessive autophagy is also shown to promote cell death following treatments with specific chemotherapeutic agents or radiotherapy, either by enhancing the induction of apoptosis or mediating 'autophagic cell death'. 37 In agreement, we found here that MKK3 depletion induced autophagic cell death, as assessed by LC3 stabilization and p62 degradation, and that blocking autophagy either with CQ or ATG5 silencing significantly reduced the cell death upon MKK3 depletion. This is also in accord with previously reported data showing the detrimental effect of MAPK14/p38a inhibition on proliferation and survival of colorectal cancer cells, leading to cell cycle arrest and autophagy-mediated cell death. ${ }^{10}$
Successful cancer eradication often needs the combination of different anticancer strategies to overcome chemoresistance and/or improve chemosensitivity. To explore whether MKK3 depletion might impact on tumor cell response to anticancer drugs, we demonstrated that MKK3 knockdown improves response to therapies, in both wtp53 and mutp53 cancer cells, allowing chemotherapeutic dose reduction.

Interestingly, we found that MKK3 deficiency induced, in wtp53-bearing cancer cells, an evident stabilization of p53 protein level that was straightly linked to ER stress induction, according to recent data. ${ }^{38}$ However, p53 stabilization does not contribute in p21 gene expression and authophagy as its depletion does not impact on the biological effects observed upon MKK3 depletion (Figures $2 \mathrm{~d}$ and e and data not shown). 
a

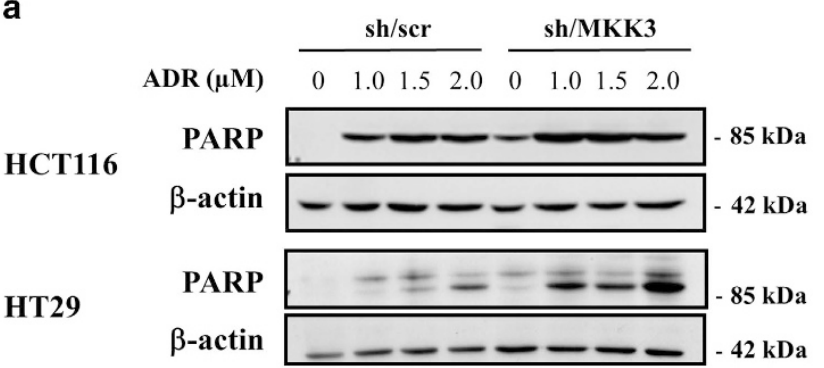

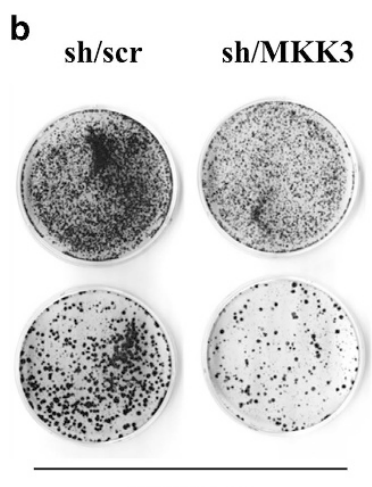

HCT116

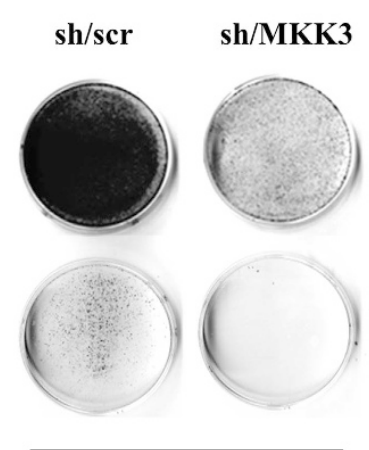

MDA-MB468

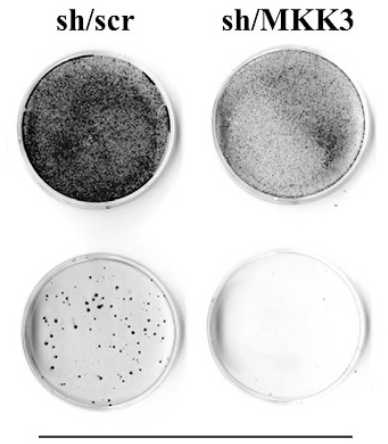

HT29
CT

ADR

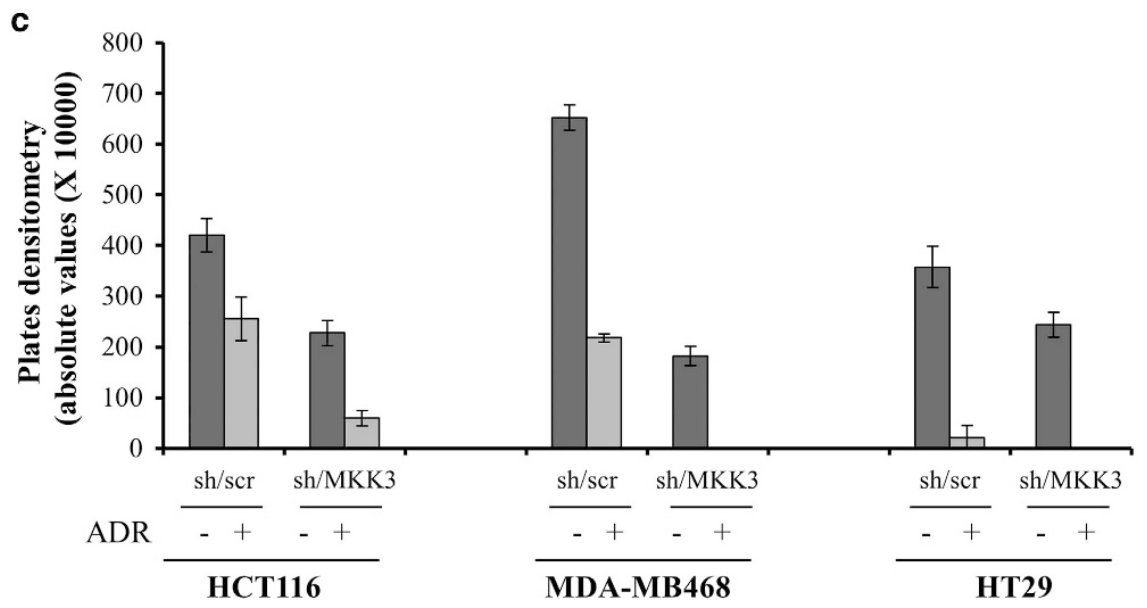

Figure 5 MKK3 depletion increases chemotherapeutic response in both wtp53 and mutp53 cancer cells. (a) Engineered sh/scr and sh/MKK3 HCT116 and HT29 cell lines were maintained in DOX condition for $48 \mathrm{~h}$ and treated/untreated for $24 \mathrm{~h}$ with $\operatorname{ADR}(1,1.5$, and $2 \mu \mathrm{M})$. Cells were then collected and protein lysates were analyzed by western blot analysis. The membrane was probed with specific anti-PARP and anti- $\beta$-actin (as loading control) antibodies. (b) Clonogenic survival assay of: HCT116 (left panel), MDA-MB468 (middle panel), and HT29 (right panel) sh/scr and sh/MKK3 cell lines, DOX-induced for $48 \mathrm{~h}$ and then treated with ADR $0.1 \mu \mathrm{M}$ (HCT116 and MDA-MB468) or $0.5 \mu \mathrm{M}$ (HT29) for $24 \mathrm{~h}$. After treatment, the culture medium was replenished, and cells were maintained at $37^{\circ} \mathrm{C}$ for 14 days. Grown colonies were stained with crystal violet. (c) Densitometric analyses of clonogenic assays as described in $\mathbf{b}$. Plates were scanned and quantified by the ImageJ software. The graph shows the absolute values of plates densitometry. Plating was performed in triplicate

In conclusion, our study demonstrates that MKK3 targeting has an important effect in reducing tumor cells proliferation and survival, both in vitro and in vivo, without affecting normal cells. Noteworthy, MKK3 depletion showed a potential additive effect with chemotherapeutic drugs on reducing tumor growth, likely through different triggered mechanisms. These data suggest the potential use of MKK3 inhibitors as an adjuvant therapy to potentiate the efficiency of chemotherapies in nonresponder patients, although further studies will be necessary to confirm our hypothesis.

\section{Materials and Methods}

Cell lines. The human lines HCT116 (colorectal carcinoma), ${ }^{39}$ FB1329 (human fibroblast), ${ }^{40} \mathrm{H} 1299$ (non-small-cell lung carcinoma), ${ }^{9}$ MDA-MB468 (breast adenocarcinoma), ${ }^{9}$ and engineered HT29 (colon adenocarcinoma)-sh/scr and -sh/MKK3 sublines ${ }^{9}$ were cultured in Dulbecco's modified Eagle's medium (Eurobio, Les Ulis, France), whereas the MCF7 line (breast adenocarcinoma) ${ }^{41}$ was cultured in Dulbecco's modified Eagle's medium-F12 (1:1). All tissue culture media were supplemented with $10 \%$ fetal bovine serum (GIBCO-BRL, Grand Island, NY, USA), L-glutamine (2 mM), and Penicillin/Streptomycin (100 U/ml; Life Technologies Inc., Eggenstein, Germany). The MCF10A line (normal breast epithelial; kindly provided from $\operatorname{Dr} S$ Anastasi) was cultured in MEGM supplemented with bovine pituitary extract $(52 \mu \mathrm{g} / \mathrm{ml})$, hydrocortisone $(0.5 \mu \mathrm{g} / \mathrm{ml})$, hEGF $(10 \mathrm{ng} / \mathrm{ml})$, and insulin 


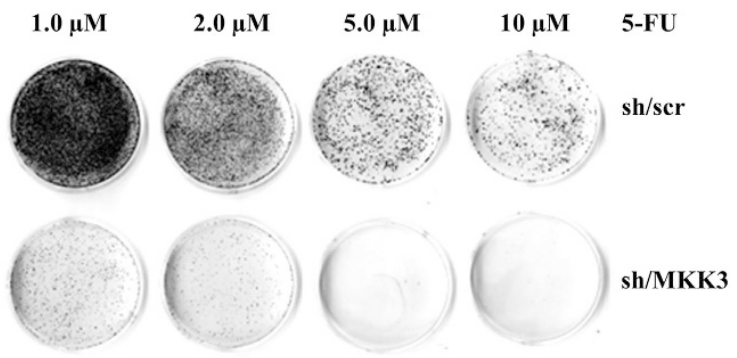

b

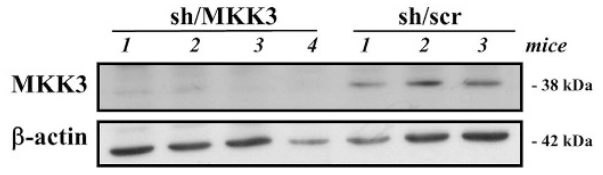

C

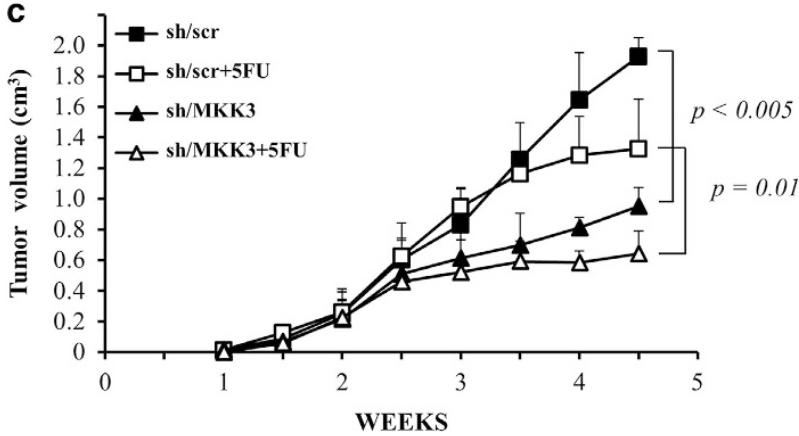

Figure 6 MKK3 depletion affects xenograft tumor growth and increases chemotherapeutic response in vivo. (a) Dose-response effect of 5-FU on clonogenic survival in HT29-sh/scr and sh/MKK3 cell lines, DOXI-induced for $48 \mathrm{~h}$, and then treated with 5-FU $(1,2,5$, and $10 \mu \mathrm{M})$ for $24 \mathrm{~h}$. After treatment, the culture medium was replenished, and cells were maintained at $37^{\circ} \mathrm{C}$ for 14 days. Grown colonies were stained with crystal violet. (b) Efficient MKK3 depletion was achieved in vivo, assessed by western blot analysis performed on a representative number of xenograft tumors generated with sh/scr and sh/MKK3 engineered HT29 cancer cells injected in nude mice (CD1/SWISS). Numbers identify single animal. (c) After tumor nodule formation, DOX (2.0 g/l, tap water) was delivered to all mice. To assess chemotherapeutic response in MKK3-depleted tumors, 5 -FU $(50 \mathrm{mg} / \mathrm{kg}$, intraperitoneal) was delivered to a subgroup of sh/scr and sh/MKK3 tumor-bearing mice (8 mice/group). Tumor growth was followed by calliper measurements twice a week. Representative data of two independent experiments are reported. Student's $t$-test analyses were performed to assess significance between sh/scr and sh/MKK3 tumorbearing mice $(P<0.05)$ and along with 5 -FU treatments $(P=0.01)$

( $5 \mu \mathrm{g} / \mathrm{ml}$; MEGM Bullet Kit, Lonza Corporation, Walkersville, MD, USA). All lines were grown at $37^{\circ} \mathrm{C}$ in a humidified atmosphere with $5 \% \mathrm{CO}_{2}$.

Lentiviral infection. HCT116, MCF7, MDA-MB468, H1299, FB1329, and MCF10A lines have been engineered with a lentiviral-based TET-OFF inducible RNA interference system carrying shRNA sequences specific to human MKK3 (sh/MKK3) or control scrambled (sh-scr) as previously described..$^{9}$ To induce shRNA expression, all engineered -sh/scr and -sh/MKK3 sublines were challenged with DOX (Sigma-Aldrich, St. Louis, MO, USA; $1 \mu \mathrm{g} / \mathrm{ml}$ ), added to the culture medium after seeding and freshly added every 3 days.

Transfections. siRNAs specific for human ATG5 as well as siRNA unrelated to the human genome were purchased from Dharmacon (Thermo Scientific, Milan, Italy) and delivered to cells as elsewhere described. ${ }^{27}$

To deplete endogenous wtp53 in HCT116-sh/MKK3 sublines, cells were transfected with pRetroSuper vector carrying sh/RNA specific to $\mathrm{p} 53(\mathrm{sh} / \mathrm{p} 53)^{42}$ or control (sh/RNA). Transfections were performed with Lipofectamine/Plus reagent (Invitrogen, Monza, Italy) following manufacture's instruction. Then, $48 \mathrm{~h}$ post transfection, cells were selected with Puromycin ( $2 \mu \mathrm{g} / \mathrm{ml}$; Sigma-Aldrich) to generate mixed population.

Cell proliferation and survival analyses. Cells were seeded $\left(2.0 \times 10^{4} /\right.$ 6 -well plates) and induced with DOX $(1 \mu \mathrm{g} / \mathrm{ml})$. After $48 \mathrm{~h}$, in a time-coursedependent manner, both floating and adherent cells were collected, stained with $0.4 \%$ Trypan blue reagent (Sigma, St. Louis, MO, USA), and counted to determine cell proliferation and viability with hemocytometer. All the experiments were performed in triplicate.

To determine the percentage of apoptotic cell death along with ATG5 depletion, $25 \mu \mathrm{l}$ of cell suspensions were mixed with $1 \mu \mathrm{l}$ of dye mix (DAPI, $1 \mu \mathrm{g} / \mathrm{ml}+$ ethidium bromide, $100 \mu \mathrm{g} / \mathrm{ml}$ ). The mixture was placed on a microscope slide, covered with a 22- $\mathrm{mm}^{2}$ coverslip and slides examined with 'Zeiss Axioskop 2 plus' fluorescent microscope. For each sample, 200 cells were counted and recorded as V (viable cells), NVN (non-viable cells with normal nuclei), and NVA (non-viable cells with apoptotic nuclei) characterized by highly condensed or fragmented nuclei. The $\%$ of apoptotic dead cells was then calculated as follow: \% apoptotic cell $=100 \times$ NVA/ $(\mathrm{VA}+\mathrm{NVN}+\mathrm{NVA})$.

Semi-quantitative reverse transcriptase-PCR. Cells were seeded $\left(1.5 \times 10^{5} / 60 \mathrm{~mm}\right.$ dish) and induced with DOX $(1 \mu \mathrm{g} / \mathrm{ml})$ for $72 \mathrm{~h}$, unless otherwise indicated. Total RNAs, extracted with TRIzol (15596-026; Invitrogen), were retrotranscribed with Moloney-Murine-Leukemia virus reverse transcriptase (M-MLV-RT, Invitrogen) following the manufacturer's instruction. For semi-quantitative PCR, cDNAs were amplified by Hot-Master Taq (5PRIME) with specific set of primers: hGAPDH (FOR5'-ATGACATCAAGAACGTGGTG-3', REV5'-CATACCAGGAAATGA GCTTG-3'); hMKK3 (FOR5'- GTGGAGCCCGCAGTCCTCTA-3', REV5'-GGGTGG CTTGGACATGCAG-3'); hp21 (FOR5'-CCCCTTCGGCCCGGTGGAC-3', REV5'CCGTTTTCGACCCTGAGAG-3'); hp53 (FOR5'-GTCTGGGCTTCTTGCATTCT-3', REV5'-AATCAACCCACAGCTGCAC-3'); and hCHOP (FOR5'- GCACCTCCCA GAGCCCTCACTCTCC-3', RE5'-GTCTACTCCAAGCCTTCCCCCTGCG-3').

Western blotting. Cells $\left(1.5 \times 10^{5}\right.$ cells $/ 60 \mathrm{~mm}$ dishes $)$ were washed twice in ice-cold PBS, harvested by scraping, and then lysed in $1 \times$ RIPA buffer $(150 \mathrm{~mm}$ $\mathrm{NaCl}, 1 \%$ Triton X-100, 0.25\% sodium deoxycholate, $0.1 \%$ SDS, $50 \mathrm{~mm}$ Tris $/ \mathrm{HCl}$, $\mathrm{pH} 8.0$, and $20 \mathrm{~mm}$ EDTA) supplemented with $1 \times$ protease and phosphatase inhibitor mixture (Sigma-Aldrich), $1 \mathrm{~mm}$ phenylmethylsulfonyl fluoride (SigmaAldrich), $50 \mathrm{~mm}$ sodium fluoride (Sigma), and $50 \mathrm{~mm}$ dithiothreitol (Bio-Rad, Hercules, CA, USA). Lysates were incubated for $30 \mathrm{~min}$ in ice, clarified by centrifugation, and resolved onto 10 or $18 \%$ SDS-polyacrylamide gel electrophoresis; $30 \mu \mathrm{g} /$ lane). Blotting was performed according to the standard protocols, and filters were immuno-reacted with the following antibodies: rabbit monoclonal antiMKK3 (D4C3; 1: 1000; Cell Signaling), mouse anti-p53 (DOI), ${ }^{43}$ rabbit polyclonal anti-Phospho-p53 (Ser392; 1: 1000; Ser51; Cell Signaling), mouse anti-actin (Ab-1; Calbiochem, San Diego, CA, USA), and rabbit anti-p21 (Santa Cruz Biotechnology, Dallas, TX, USA); rabbit polyclonal anti-LC3 (1:1000; Sigma-Aldrich), mouse monoclonal anti-p62 (SQSTM1; 1: 1000; Santa Cruz Biotechnology, Dallas, TX, USA), rabbit monoclonal anti-GRP78/BiP (C50B12; 1:1000; Cell Signaling), rabbit monoclonal anti-IRE1 $\alpha$ (14C10; $1: 1000$; Cell Signaling), rabbit polyclonal anti-Phospho-EIF2 $\alpha$ (pEIF2 $\alpha ; 1: 1000$; Ser51; Cell Signaling), rabbit polyclonal antiEIF2 $\alpha$ (1: 1000; Cell Signaling), rabbit polyclonal anti-cleaved Caspase-3 (Asp175; 1: 1000; Cell Signaling), rabbit polyclonal anti-ATG5 (1:1000; Sigma-Aldrich), and rabbit monoclonal anti-PARP (Poly-ADP-ribose polymerase) p89 Fragment (Asp214; 1:1000; Cell Signaling). Secondary HRP-conjugated anti-mouse or anti-rabbit (Bio-Rad) antibodies were used. Detection of immuno-reactions was performed by ECL kit (Amersham Biosciences, Glattbrugg, Switzerland). Images were acquired with the EPSON Expression $10000 \mathrm{XL}$ scanner (Epson, Long Beach, CA, USA) and densitometry was performed with the ImageJ software (NIH, Bethesda, MD, USA).

Chemical autophagy inhibition. MCF7-sh/scr and -sh/MKK3 sublines were seeded $\left(1.5 \times 10^{5}\right.$ cells $/ 60 \mathrm{~mm}$ dishes $)$ and supplemented with DOX. Then, $24 \mathrm{~h}$ later, cells were treated/untreated with $\mathrm{CQ}(25 \mu \mathrm{M}$, Sigma-Aldrich), and collected subsequently at $96 \mathrm{~h}$ of total DOX induction for cells viability analyses. To inhibit autophagy in MDA-MB468-sh/scr and -sh/MKK3 sublines, cells were plated $\left(1.5 \times 10^{5}\right.$ cells $/ 60 \mathrm{~mm}$ dishes $)$ and supplemented with DOX. Seventy-two hours later cells were treated/untreated with $\mathrm{CQ}(25 \mu \mathrm{M})$, and collected for western blot analyses $48 \mathrm{~h}$ later. 
Chemotherapeutic treatments. To study apoptotic response, HCT116- and HT29-sh/scr and sh/MKK3 sublines were plated $\left(1.5 \times 10^{5}\right.$ cells/60 mm dishes) and supplemented with DOX. Fourth-eight hours later, cells were challenged with Adriamycin/Doxorubicin (ADR; Pharmacia, Milan, Italy) and collected $24 \mathrm{~h}$ later for western blot analyses.

For clonogenic survival assay, engineered -sh/scr and -sh/MKK3 sublines were seeded along with DOX as follow: HCT116 $\left(4.0 \times 10^{4}\right.$ cells $/ 60 \mathrm{~mm}$ dishes), MDAMB468, and HT29 $\left(1.5 \times 10^{5}\right.$ cells/60 mm dishes). Fourthly-eight hours later, cells were challenged with either $\operatorname{ADR}(0.1-0.5 \mu \mathrm{M})$ or 5 -FU $(1,2,5$, and $10 \mu \mathrm{M}$; Roche, Milan, Italy). Then, $24 \mathrm{~h}$ later, cells were washed three times with PBS and fed with complete medium supplemented with DOX, which was replenished every $72 \mathrm{~h}$. Fourteen days later, colonies were stained with crystal violet and analyzed by densitometry with ImageJ software. Experiments were performed in triplicate and repeated three times.

In vivo assay. Exponentially growing HT29-sh/scr and sh/MKK3 sublines were injected $\left(5 \times 10^{5} \mathrm{cell} / \mathrm{mouse}\right)$ subcutaneously in 45 -day-old female nude mice (CD1/Swiss, Charles River, Lecco, Italy). Two weeks later, which tumors reached a volume of $0.2 \mathrm{~cm}^{3}$, all mice were delivered with DOX $(2.0 \mathrm{~g} / /)$ as reported. ${ }^{43}$ Animals bearing HT29-sh/scr or sh/MKK3 tumors were randomly subdivided into groups (8 mice/group), and either treated or untreated with $5-\mathrm{FU}(50 \mathrm{mg} / \mathrm{kg})$ by intraperitoneal injection at days 7, 9, 11 after DOX delivering started. Tumor growth was followed by caliper measurements twice a week and tumor volumes (TV) estimated by the formula: TV $=a \times\left(b^{2}\right) / 2$, where $a$ and $b$ are tumor length and width, respectively. At the end of the experiment, all the animals were killed, tumors excised and analyses by western blot analysis to ascertain the occurred MKK3 depletion in vivo. All the procedures involving animals and their care were approved by the Ethical Committee of the Regina Elena Cancer Institute (CE/532/12) and were conformed to the relevant regulatory standards in accordance with the Italian legislation.

Statistical analysis. All experiments were performed in triplicate. Numerical data are reported as means \pm S.D.s. Student's $t$-test was used for statistical significance of the differences between treatment groups. Statistical analysis was performed using analysis of variance at $5 \%(P<0.05)$ or $1 \%$ $(P<0.01)$.

\section{Conflict of Interest}

The authors declare no conflict of interest.

Acknowledgements. We thank Dr. Fanciulli for kindly providing $h \mathrm{CHOP}$ primers, anti-IRE $1 \alpha$, and anti-GRP78 antibodies. We thank Dr. Bruno for technical advices. This work was supported by grants from the Associazione Italiana per la Ricerca sul Cancro (AIRC) to GB (IG \#8804) and GD (IG\#11377).

1. Johnson GL, Lapadat R. Mitogen-activated protein kinase pathways mediated by ERK, JNK, and p38 protein kinases. Science 2002; 298: 1911-1912.

2. Raingeaud J, Whitmarsh AJ, Barrett T, Derijard B, Davis RJ. MKK3- and MKK6-regulated gene expression is mediated by the $\mathrm{p} 38$ mitogen-activated protein kinase signal transduction pathway. Mol Cell Biol 1996; 16: 1247-1255.

3. Han J, Lee JD, Jiang Y, Li Z, Feng L, Ulevitch RJ et al. Characterization of the structure and function of a novel MAP kinase kinase (MKK6). J Biol Chem 1996; 271 : 2886-2891.

4. Raingeaud J, Gupta S, Rogers JS, Dickens M, Han J, Ulevitch RJ et al. Pro-inflammatory cytokines and environmental stress cause p38 mitogen-activated protein kinase activation by dual phosphorylation on tyrosine and threonine. J Biol Chem 1995; 270: 7420-7426.

5. Pearson G, Robinson F, Beers Gibson T, Xu BE, Karandikar M, Berman K et al. Mitogenactivated protein (MAP) kinase pathways: regulation and physiological functions. Endocr Rev 2001; 22: 153-183.

6. Dérijard B, Raingeaud J, Barrett T, Wu IH, Han J, Ulevitch RJ et al. Independent human MAP-kinase signal transduction pathways defined by MEK and MKK isoforms. Science 1995; 267: 682-685.

7. Sluss HK, Barrett T, Dérijard B, Davis RJ. Signal transduction by tumor necrosis factor mediated by JNK protein kinases. Mol Cell Biol 1994; 14: 8376-8384.

8. Demuth T, Reavie LB, Rennert JL, Nakada S, Hoelzinger DB, Beaudry CE et al. MAP-ing glioma invasion: mitogen-activated protein kinase kinase 3 and p38 drive glioma invasion and progression and predict patient survival. Mol. Cancer Ther 2007; 6: 1212-1222.
9. Gurtner A, Starace G, Norelli G, Piaggio G, Sacchi A, Bossi G et al. Mutp53-induced up-regulation of mitogen-activated protein kinase kinase 3 contributes to gain of function. J Biol Chem 2010; 285: 14160-14169.

10. Chiacchiera F, Simone C. Signal-dependent regulation of gene expression as a target for cancer treatment: inhibiting p38alpha in colorectal tumors. Cancer Lett 2008; 265: $16-26$.

11. Mizushima N, Levine B, Cuervo AM, Klionsky DJ. Autophagy fights disease through cellular self-digestion. Nature 2008; 451: 1069-1075.

12. Levine B, Klionsky DJ. Development by self-digestion: molecular mechanisms and biological functions of autophagy. Dev Cell 2004; 6: 463-477.

13. Klionsky DJ. Autophagy: from phenomenology to molecular understanding in less than a decade. Nat Rev Mol Cell Biol 2007; 8: 931-937.

14. Ohsumi Y. Molecular dissection of autophagy: two ubiquitin-like systems. Nat Rev Mol Cell Biol 2001; 2: 211-216

15. Levine B, Kroemer G. Autophagy in the pathogenesis of disease. Cell 2008; 132 $27-42$.

16. Yang Z, Klionsky DJ. Eaten alive: a history of macroautophagy. Nat Cell Biol 2010; 12 : 814-822.

17. Klionsky DJ, Abdalla FC, Abeliovich H, Abraham RT, Acevedo-Arozena A, Adeli K et al. Guidelines for the use and interpretation of assays for monitoring autophagy. Autophagy 2012; 8: 445-544.

18. Sui X, Jin L, Huang X, Geng S, He C, Hu X et al. p53 signaling and autophagy in cancer: a revolutionary strategy could be developed for cancer treatment. Autophagy 2011; 7 : 565-571.

19. Yang Z, Klionsky DJ. An overview of the molecular mechanism of autophagy. Curr Top Microbiol Immunol 2009; 335: 1-32.

20. Lum JJ, DeBerardinis RJ, Thompson CB. Autophagy in metazoans: cell survival in the land of plenty. Nat Rev Mol Cell Biol 2005; 6: 439-448.

21. Boya P, Gonzalez-Polo RA, Casares N, Perfettini JL, Dessen P, Larochette N et al. Inhibition of macroautophagy triggers apoptosis. Mol Cell Biol 2005; 25: 1025-1040.

22. Kroemer G, Galluzzi L, Vandenabeele P, Abrams J, Alnemri ES, Baehrecke EH et al. Classification of cell death: recommendations of the Nomenclature Committee on Cell Death 2009. Cell Death Differ 2009; 16: 3-11.

23. Platini F, Perez-Tomas R, Ambrosio S, Tessitore L. Understanding autophagy in cell death control. Curr Pharm Des 2010; 16: 101-113.

24. Rodriguez OC, Choudhury S, Kolukula V, Vietsch EE, Catania J, Preet A et al. Dietary downregulation of mutant p53 levels via glucose restriction: mechanisms and implications for tumor therapy. Cell Cycle 2012; 11: 4436-4446.

25. Ogata M, Hino S, Saito A, Morikawa K, Kondo S, Kanemoto S et al. Autophagy is activated for cell survival after endoplasmic reticulum stress. Mol Cell Biol 2006; 26: 9220-9231.

26. Kouroku Y, Fujita E, Tanida I, Ueno T, Isoai A, Kumagai $\mathrm{H}$ et al. ER stress (PERK/elF2alpha phosphorylation) mediates the polyglutamine-induced LC3 conversion, an essential step for autophagy formation. Cell Death Differ 2007; 14: 230-239.

27. Garufi A, Pucci D, D'Orazi V, Cirone M, Bossi G, Avantaggiati ML et al. Degradation of mutant $\mathrm{p} 53 \mathrm{H} 175$ protein by $\mathrm{Zn}(\mathrm{II})$ through autophagy. Cell Death Dis 2014; 5: e1271.

28. Afford S, Randhawa S. Apoptosis. Mol Pathol 2000; 53: 55-63.

29. Klionsky DJ, Abeliovich H, Agostinis P, Agrawal DK, Aliev G, Askew DS et al. Guidelines for the use and interpretation of assays for monitoring autophagy in higher eukaryotes. Autophagy 2008; 4: 151-175.

30. Poole B, Ohkuma S. Effect of weak bases on the intralysosomal pH in mouse peritoneal macrophages. J Cell Biol 1981; 90: 665-669.

31. Marciniak SJ, Yun CY, Oyadomari S, Novoa I, Zhang $Y$, Jungreis $R$ et al. CHOP induces death by promoting protein synthesis and oxidation in the stressed endoplasmic reticulum. Genes Dev 2004; 18: 3066-3077.

32. Mizushima N, Yoshimori T, Ohsumi Y. The role of Atg proteins in autophagosome formation Annu Rev Cell Dev Biol 2011; 27: 107-132.

33. Loehrer PJ Sr, Turner S, Kubilis P, Hui S, Correa J, Ansari R et al. A prospective randomized trial of fluorouracil versus fluorouracil plus cisplatin in the treatment of metastatic colorectal cancer: a Hoosier Oncology Group trial. J Clin Oncol 1988; 6 : 642-648.

34. Kondo $\mathrm{Y}$, Kanzawa $\mathrm{T}$, Sawaya $\mathrm{R}$, Kondo $\mathrm{S}$. The role of autophagy in cancer development and response to therapy. Nat Rev Cancer 2005; 5: 726-734.

35. Janku F, McConkey DJ, Hong DS, Kurzrock R. Autophagy as a target for anticancer therapy. Nat Rev Clin Oncol 2011; 8: 528-539.

36. Garufi A, Ricci A, Trisciuoglio D, lorio E, Carpinelli G, Pistritto G et al. Glucose restriction induces cell death in parental but not in HIPK2 depleted RKO colon cancer cells: molecular mechanisms and implications for tumor therapy. Cell Death Dis 2013; 4: e639.

37. Choi KS. Autophagy and cancer. Exp Mol Med 2012; 44: 109-120.

38. Li J, Lee B, Lee AS. Endoplasmic reticulum stress-induced apoptosis: multiple pathways and activation of p53-up-regulated modulator of apoptosis (PUMA) and NOXA by p53. J Biol Chem 2006; 281: 7260-7270.

39. Strigari L, Mancuso M, Ubertini V, Soriani A, Giardullo P, Benassi M et al. Abscopal effect of radiation therapy: Interplay between radiation dose and p53 status. Int J Radiat Bio/ 2014; 90 : 248-255. 
40. Falcone G, Mazzola A, Michelini F, Bossi G, Censi F, Biferi MG et al. Cytogenetic analysis of human cells reveals specific patterns of DNA damage in replicative and oncogene-induced senescence. Aging Cell 2013; 12: 312-315.

41. Ubertini V, Norelli G, D'Arcangelo D, Gurtner A, Cesareo E, Baldari S et al. Mutant p53 gains new function in promoting inflammatory signals by repression of the secreted interleukin-1 receptor antagonist. Oncogene 2014; e-pub ahead of print 7 July 2014; doi:10.1038/onc.2014.191.

42. Bossi G, Lapi E, Strano S, Rinaldo C, Blandino G, Sacchi A et al. Mutant p53 gain of function: reduction of tumor malignancy of human cancer cell lines through abrogation of mutant p53 expression. Oncogene 2006; 25: 304-309.

43. Bossi G, Marampon F, Maor-Aloni R, Zani B, Rotter V, Oren M et al. Conditional RNA interference in vivo to study mutp53 oncogenic gain of function on tumor malignancy. Cell Cycle 2008; 7: 1870-1879. (c) (i) Cell Death and Disease is an open-access journal published by Nature Publishing Group. This work is licensed under a Creative Commons Attribution 4.0 International Licence. The images or other third party material in this article are included in the article's Creative Commons licence, unless indicated otherwise in the credit line; if the material is not included under the Creative Commons licence, users will need to obtain permission from the licence holder to reproduce the material. To view a copy of this licence, visit http://creativecommons.org/ licenses/by/4.0 